Submitted:

27.08.2019

Accepted:

11.09.2019

Published:

30.09.2019

Keywords

stress urinary incontinence, ultrasonography,

transvaginal transducer,

urethral funneling, suburethral sling

\section{The role of TVT position in relation to the pubic symphysis in eliminating the symptoms of stress urinary incontinence and urethral funneling}

\author{
Piotr Pędraszewski ${ }^{1}$, Edyta Wlaźlak², Wiktor Wlaźlak², Magda Krzycka², \\ Paulina Pająk², Grzegorz Surkont²
}

${ }^{1}$ Department of Gynecology and Obstetrics, Provincial Integrated Hospital in Płock, Płock, Poland

${ }^{2}$ First Department of Gynecology and Obstetrics, Clinic for Gynecological Surgery and Oncology, Medical University of Lodz, Lodz, Poland

Correspondence: Wlaźlak Edyta, First Department of Gynecology and Obstetrics, Medical University of Lodz, Clinic for Gynecological Surgery and Oncology, Wileńska 37, 94-029 Lodz, Poland; tel.: +48 502626675, e-mail: edytawlazlak@gmail.com

DOI: $10.15557 / \mathrm{JoU} .2019 .0031$

\begin{abstract}
The introduction of suburethral sling was a breakthrough in the treatment of stress urinary incontinence in women. The method is highly effective. However, the mechanism of action of a sling and the reasons for surgical failures are not fully understood. Aim: The aim of the study was to assess the impact of sling-pubic symphysis distance on eliminating the symptoms of stress urinary incontinence and urethral funneling. Materials and methods: The analysis included 106 patients who reported 3 to 6 months after sling placement for a follow-up visit encompassing clinical examination and standard ultrasonography. We evaluated the position of sling in relation to the pubic symphysis, urethral length, as well as urethral funneling length and width. Results: Cure criteria were met by 91 patients. Elimination of urethral funneling was achieved in $76.9 \%(n=70)$ of cured patients. Urethral funneling was still present, yet shorter by a mean of $10.2 \mathrm{~mm}(p=0.02)$ than before surgery in the remaining cured patients. There was a $32.5 \%$ reduction in the mean relative length of urethral funneling $(p=0.002)$. No significant differences were found in the pre- and postoperative funneling width. The tapepubic symphysis distance was lower in cured women: $23.2 \mathrm{~mm}$ vs. $26.1 \mathrm{~mm}$ in failed women $(p=0.04)$. Similar observations were made for cured patients with persistent urethral funneling vs. failed patients ( $22.47 \mathrm{~mm}$ vs. $26.0 \mathrm{~mm}, p=0.027)$. There were no differences between cured patients without urethral funneling and cured patients with persistent postoperative funneling (23.5 mm; $22.5 \mathrm{~mm} ; p=0.417)$. Conclusions: Tape position in relation to the pubic symphysis is important for the elimination of stress urinary incontinence. Sling location closer to the pubic symphysis reduces the length of urethral funneling, but has no effects on its width
\end{abstract} in cured patients with persistent postoperative funneling. 


\section{Introduction}

Stress urinary incontinence (SUI) is the most common type of urinary incontinence (UI) in women ${ }^{(1,2)}$. However, its pathophysiology is not fully understood. The symptoms of SUI are assumed to be associated with urethral hypermobility and low maximum urethral closure pressure in most $\operatorname{cases}^{(3,4)}$

The introduction of tension-free vaginal tape (TVT) was a breakthrough in the surgical treatment of SUI. The method is highly effective, with cure rates up to $93 \%$ at 6 months after procedure ${ }^{(5)}$. However, the exact mechanism of TVT and the causes of surgical failures remain unknown. Ultrasound findings indicate two possible mechanisms during increased intra-abdominal pressure: urethral compression against the pubic symphysis ${ }^{(6)}$ and urethral compression by the tape ${ }^{(5)}$.

In addition to standard diagnostic methods, such as cough stress test, pad test, voiding diary or urodynamic testing, ultrasonography is also likely to play an important role in the diagnosis and assessment of patients with $\mathrm{SUI}^{(2)}$. Pelvic floor ultrasound performed with transvaginal probe (PFS-TV) shows excellent and good repeatability of the obtained results ${ }^{(4,7,8)}$. In addition to sonographic evaluation of urethral and bladder neck mobility, as well as postoperative sonographic localization of the tape, visualization of urethral funneling, which, according to different authors, may overlap with SUI in $18.6 \%$ to $100 \%$ women, is also possible ${ }^{(2,9)}$. Sonographic assessment of urethral funneling may be an important diagnostic parameter in SUI and help monitor surgical outcomes in SUI patients in the future ${ }^{(2)}$. However, the data published so far is too sparse.

Previous studies indicate that a tape positioned closer to the pubic symphysis increases the chances for successful elimination of $\mathbf{S U I}^{(6)}$. So far, it has not been verified whether the distance between the tape and the pubic symphysis has any effects on the elimination of urethral funneling.

The aim of the study was to assess the impact of tape-pubic symphysis distance on the elimination of SUI symptoms and urethral funneling.

\section{Materials and methods}

A total of 121 patients were qualified for TVT procedure between 2006 and 2012 (Ethicon, Johnson\&Johnson, USA). Of these, 115 women reported for the procedure. All patients gave their consent to participate in the study. Treatment outcomes of 106 patients who reported for a follow-up visit within 3 to 6 months of surgery were included in the analysis. The study was approved by the Ethics Committee of the Medical University of Lodz.

Preoperative SUI was confirmed during clinical and urodynamic examination, based on objective and subjective criteria described by Kociszewski et al. ${ }^{(10,11)}$ Clinical assessment of vaginal wall prolapse was performed using the POP-Q scale $^{(12)}$. PFS-TV was performed in all patients.

The planned hospital stay after the surgery was 2 days. Foley catheter was removed about 24 hours after the surgery. Micturition disorders were diagnosed if $100 \mathrm{~mL}$ of residual urine remained after voiding and persisted for more than 1 day after catheter removal. The results of medical observations during hospital stay and followup findings obtained 3 to 6 months after TVT procedure were included in the analysis. Postoperative diagnosis included clinical and ultrasonographic (PFS-TV) examination $^{(10,11)}$.

Standardized PFS-TV was performed using Philips EnVisorC, Hitachi EUB-525 and BK Pro Focus ultrasound systems, a $6.5 \mathrm{MHz}$ transvaginal probe, $160^{\circ}$ beam angle, as in accordance with a technique developed by Kociszewski $^{(7,5,10,11)}$, in a patient in a semi-sitting position with a normalized bladder volume of 250-300 mL, which was calculated from 3 bladder dimensions: transverse, anteroposterior and superior-inferior.

The assessment of surgical outcomes included subjective symptoms, such as inguinal pain, urge and voiding dysfunction. The group of cured patients included women with negative cough test, negative 1 -hour pad test $(<2 \mathrm{~g})$, and with Visual Analogue Scale (VAS) score of 0 to 1 . If the patients failed to meet all these criteria, the treatment was considered unsuccessful and they were classified in the failed group.

PFS-TV was performed in patients after TVT procedure to assess tape position in relation to the pubic symphysis. The examination was started by positioning the probe in alignment with the patient's axis. A single image was taken to visualize the pubic symphysis (which was the only fixed point of reference), the urethra and the bladder neck, as well as to assess the position of the tape in relation to the pubic symphysis (Fig. 1, Fig. 2).

The probe angle was changed in accordance with Kociszewski technique for precise pre- and postoperative visualization of the urethral funneling. During maximum straining, we verified the presence of urethral funneling, measured its geometry and verified whether urine flow was visible in the ultrasound examination. Using the previously described technique(2), we measured urethral length, the funneling length and width, as well as we calculated the relative funneling length (\%). According to the previously published definition ${ }^{(2)}$, long funneling was estimated at $>50 \%$ of urethral length with sonographic evidence of urine flow. Short urethral funneling was diagnosed if no urine flow was seen in ultrasound and the funneling length was $\leq 50 \%$.

The investigated variables showed normal distribution. The Shapiro-Wilk test was used for normality analysis. The T-test was used for independent variables to compare the groups of cured vs. failed patients. Statistical analysis of pre- and postoperative data was conducted using the T-test 


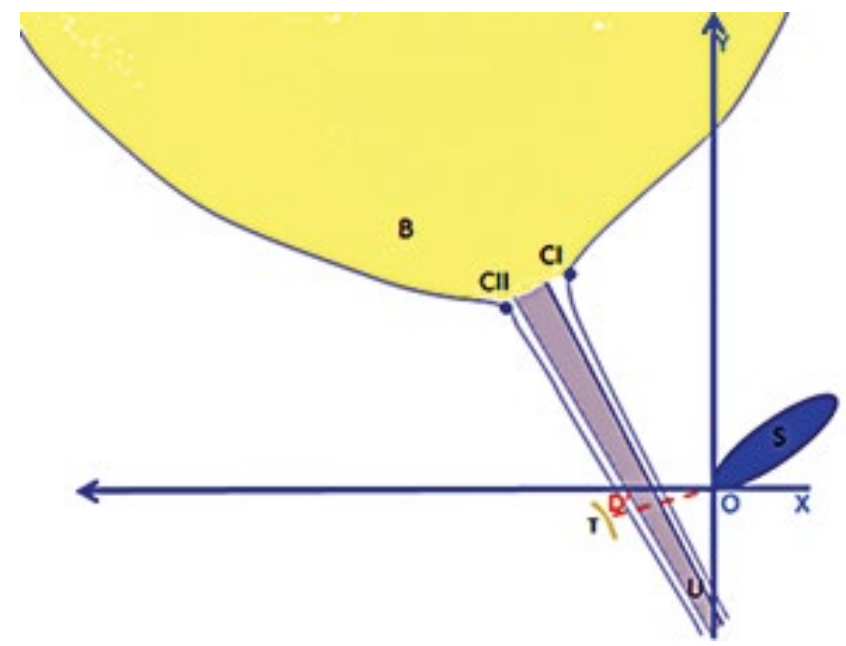

Fig. 1. Measurement scheme for the tape-pubic symphysis parameter (D'O). $S$ - pubic symphysis, $B$ - bladder, $U$ - urethra, $C I$ and CII - bladder urethral outlet, T - tape, D'O - tape-pubic symphysis parameter

for dependent variables. Mean values (with their ranges) were specified for continuous variables.

\section{Results}

A total of 106 patients were included in the analysis. The mean age of patients was 60.8 years ( 47 to 77 years). Mean BMI was $27.2 \mathrm{~kg} / \mathrm{m}^{2}$ (standard deviation $4.5 \mathrm{~kg} / \mathrm{m}^{2}$ ). A mean number of 2 childbirths (0 to 6) was reported. Natural delivery and cesarean section were reported by $83 \%$ and $13.2 \%$ of patients, respectively. Operative delivery (forceps or a vacuum extractor) was reported by $7.5 \%$ of patients. Nulligravidas accounted for 7.5\%. Patients after hysterectomy accounted for $13.2 \%(n=14)$. A total of $19.8 \%$ $(n=21)$ of patients had a history of at least one urogynecological surgery, including Burch procedure in $2.8 \%(n=3)$. There were no patients with a history of sling placement.

No significant disorders of genital statics other than second-degree rectocele in 3 women (2.8\%), who additionally underwent posterior wall repair, were found in the patients qualified for TVT procedure. Other patients underwent TVT placement only. Symptoms of dry overactive bladder were reported preoperatively by 40 women $(37.7 \%)$. There were no cases of voiding abnormalities.

A total of 106 patients reported for a follow-up visit 3 to 6 months after the surgery. No significant peri- or postoperative complications were found in any of the patients. No cases of significant post-void urine retention were observed. None of the patients developed vaginal mucosal erosion. A total of $13(12.3 \%)$ patients reported persistent symptoms of dry overactive bladder. Urge was reported by 2 patients $(1.9 \%)$. None of the patients reported de novo urge without incontinence.

On follow-up, 91 patients were considered to meet the cure criteria (group C), while 15 women were qualified as failed (group F). Figure 3 shows the number of patients qualified into different groups depending on the symptoms of SUI and urethral funneling.

Long urethral funneling was found preoperatively in all $(n=106)$ patients with the symptoms of second and third stage SUI. The relative funneling length exceeded $50 \%$ in all patients (50.2-99.7\%, mean 53.3\%); the mean absolute length was $16.7 \mathrm{~mm}(9.7-32.8 \mathrm{~mm})$. The mean funneling width was $5.5 \mathrm{~mm}(1.2-16.6 \mathrm{~mm})$.

In the group of cured patients $(n=91)$, the procedure led to complete elimination of urethral funneling in $76.9 \%$ $(n=70)$. The funneling was still visible in the remaining $23.1 \%(n=21)$ of the cured patients, but with no evident urine flow in ultrasound (short funneling). It was shorter in this group of patients compared to preoperative findings: mean $10.2 \mathrm{~mm},(4.5-17.3 \mathrm{~mm}, p=0.02)$. The mean relative length of funneling was smaller than before TVT implantation: $32.5 \%(11.8-48 \%, p=0.002)$. There were no significant differences in pre- and postoperative funneling width $(5.6 \mathrm{~mm}$ vs. $5.9 \mathrm{~mm})$.

PFS-TV showed persistent long funneling in 15 failed patients. TVT placement had no significant impact on either length or width of the funneling.

Statistically significant differences $(p=0.04)$ were found in the distance between the tape and the pubic symphysis between cured (C) and failed (F) patents. Higher values were obtained for cured (C) vs. failed (F) patients. The mean value was $23.2 \mathrm{~mm}$ in the $C$ group, and $26.1 \mathrm{~mm}$ in the F group (Fig. 4).

The analysis of the distance between the tape and the pubic symphysis confirmed a statistically significant difference in this distance between cured patients with persistent post-TVT placement urethral funneling (UF+) and failed patients $(p=0.027$, mean $(\mathrm{UF}+)=22,47 \mathrm{~mm}$, mean $(\mathrm{F})$ $=26.0 \mathrm{~mm})$.

Ultrasound measurements of the position of the TVT tape in relation to the pubic symphysis showed no significant

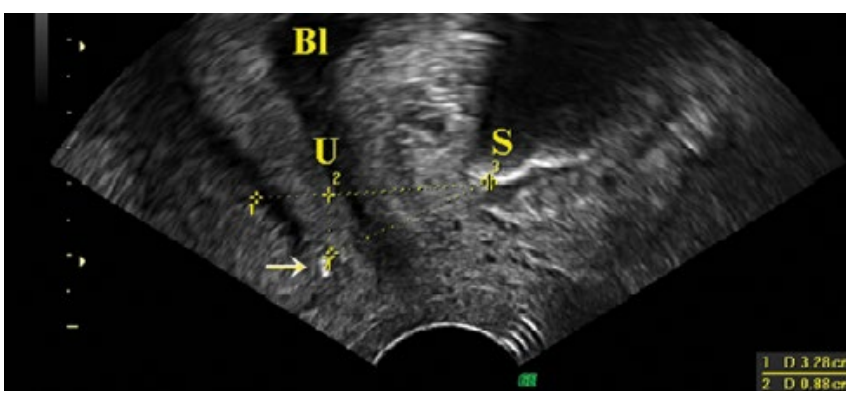

Fig. 2. Method for measuring the tape-pubic symphysis parameter (D'O) - a PFS-TV image. $S$ - pubic symphysis, $B$ - bladder, $U$ - urethra, arrow - suburethral sling, 1 - a line drawn along the lower edge of the pubic symphysis, 2 - tape position in the $Y$ axis in relation to the pubic symphysis, 3 - tape position in the $X$ axis in relation to the pubic symphysis, 4 - tape-pubic symphysis distance 


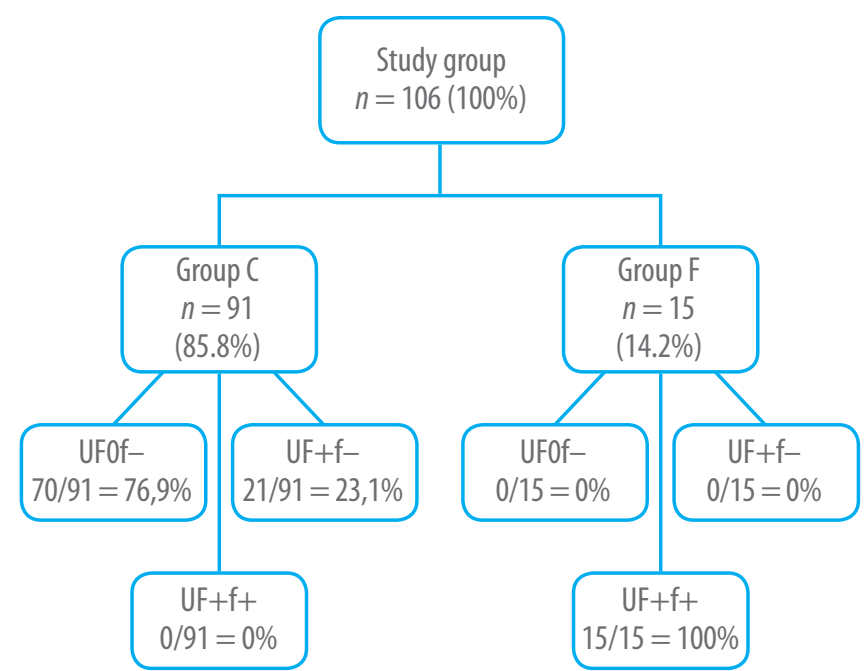

Fig. 3. Classification of patients based on postoperative symptoms of SUI and urethral funneling. C-cured, F-failed, UFOf- ultrasound: no urethral funneling, no urine flow; UF+f- ultrasound: funneling present, no urine flow (short funneling); $U F+f+-$ ultrasound: funneling present, urine flow present (long funneling)

differences between cured patients with eliminated funneling after the surgery (UF0) and cured patients with persistent postoperative funneling $(\mathrm{UF}+)(p=0.417$, mean (UF0) $=23.5 \mathrm{~mm}$, mean $(\mathrm{UF}+)=22.5 \mathrm{~mm})$.

\section{Discussion}

The analyzed results confirmed that the position of the tape in relation to the pubic symphysis has an impact on treatment outcomes in patients with SUI. According to Dietz, the sling moves within the arch around the pubic symphysis, which reduces the distance between the sling and the pubic symphysis on straining ${ }^{(6)}$. Duckett et al. pointed out that the effect of continence is achieved by urethral compression against the pubic symphysis and therefore, in their opinion, the distance between the tape and the pubic symphysis plays an important role in the successful treatment of SUI by suburethral sling placement $^{(13,14)}$.

According to Yang et al., sling tension is the only intraoperatively controllable factor. The authors used intraoperative ultrasonography for this purpose ${ }^{(15)}$.

Kociszewski et al. suggested that it is possible to modulate tape location in the longitudinal axis of the urethra by preoperative choice of the site for vaginal mucosal incision and pointed to the impact of the distance between the tape and the urethral lumen on the effectiveness of eliminating SUI after suburethral sling implantation ${ }^{(5,10,11)}$.

Dietz confirmed in his study using 4D ultrasound that the position of the tape in relation to the long and transverse urethral axis has an impact on the postoperative outcome $^{(6,16)}$
Our study did not compare the impact of sling location in relation to the pubic symphysis vs. urethra. Our findings confirm that the treatment outcome in patients with SUI depends on tape position in relation to the pubic symphysis. A tape inserted closer to the pubic symphysis offers a better chance of curing SUI. Unfortunately, surgical techniques allowing for an individually planned, optimal location in relation to the pubic symphysis are not known.

TVT positioned closer to the pubic symphysis allowed for eliminating SUI and funneling in $77 \%$ of cured patients. Our studies and analyses have never shown any significant effects of the tape-pubic symphysis distance and funneling width in cured patients with postoperatively persisting funneling or failed patients. A shorter tape-pubic symphysis distance was associated with reduced funneling length in SUI patients after TVT implantation, but had no effects in on this length in failed patients.

No analyses on the impact of surgical tape positioning in relation to the pubic symphysis on complete elimination of funneling or its size have been published in the available worldwide literature.

In our opinion, the probable causes of postoperatively persisting funneling include damaged internal urethral sphincter mechanism and periurethral structures. In the

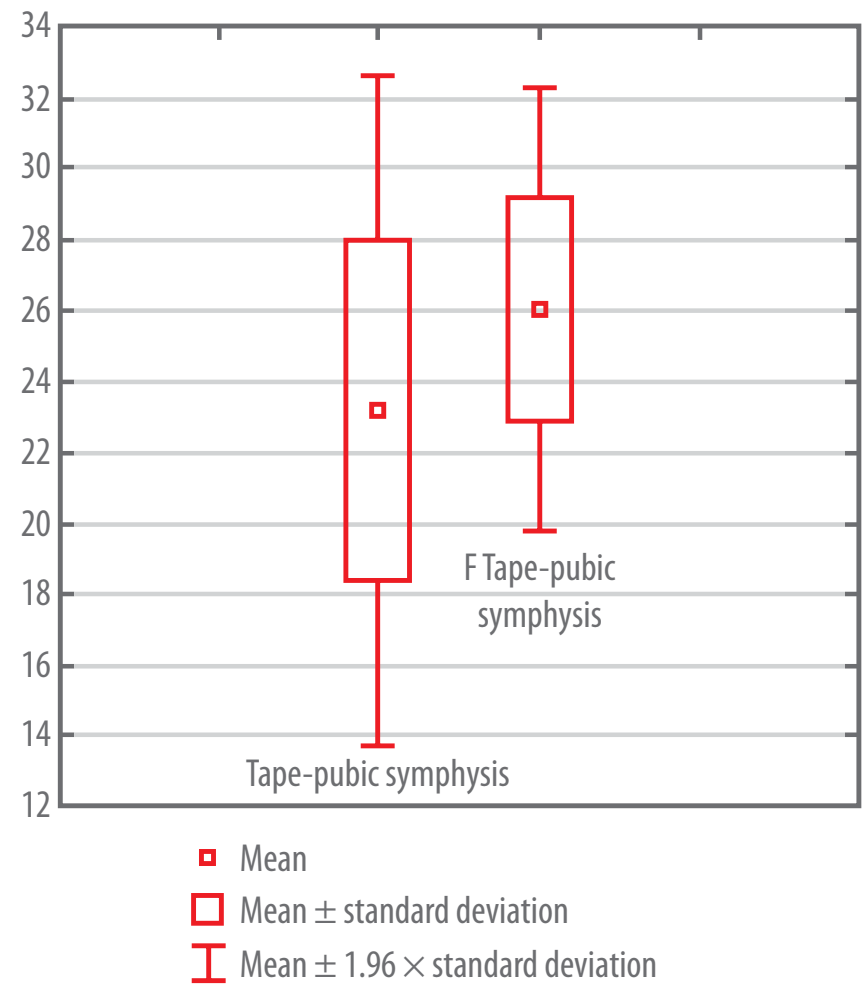

Fig. 4. Comparison of tape location parameters between cured $(C)$ and failed (F) patients. Tape-pubic symphysis - tape-pubic symphysis parameter in group $C ; F$ Tape-pubic symphysis tape-pubic symphysis parameter in group $F$ 
case of patients with efficient internal urethral sphincter mechanism, proper TVT placement may improve the effectiveness of extrasphincteric mechanism, and thus allow for complete elimination of urethral funneling.

Falconer et al. conducted a study suggesting that SUI elimination after suburethral sling placement may also result from altered metabolism of the periurethral connective tissue. The authors suspect that the tape is likely to promote restoration of connective tissue supportive properties along the sling, which is another argument for midurethral placement of the tape instead of insertion in the bladder region, where the number of periurethral fibers is significantly lower ${ }^{(17)}$.

Our findings again confirmed ${ }^{(2)}$ that a short funneling is not a symptom of SUI.

\section{References}

1. Shek KL, Chantarasorn V, Dietz HP: The urethral motion profile before and after suburethral sling placement. J Urol 2010; 183(4): 1450-1454.

2. Wlaźlak E, Kluz T, Surkont G, Kociszewski J: Urethral funneling visualized during pelvic floor sonography - analysis of occurrence among urogynecological patients. Ginekol Pol 2018; 89(2): 55-61.

3. Wlaźlak E, Surkont G, Shek KL, Dietz HP: Can we predict urinary stress incontinence by using demographic, clinical, imaging and urodynamic data? Eur J Obstet Gynecol Reprod Biol 2015; 193: 114-117.

4. Wlaźlak E, Viereck V, Kociszewski J, Kuszka A, Rautenberg O, Walser C et al.: Role of intrinsic sphincter deficiency with and without urethral hypomobility on the outcome of tape insertion. Neurourol Urodyn 2017; 36(7): 1910-1916.

5. Kociszewski J, Rautenberg O, Kuszka A, Eberhard J, Hilgers R, Viereck V: Can we place tension-free vaginal tape where it should be? The onethird rule. Ultrasound Obstet Gynecol 2012; 39(2): 210-214.

6. Dietz HP, Wilson PD: The 'iris effect': how two-dimensional and threedimensional ultrasound can help us understand anti-incontinence procedures. Ultrasound Obstet Gynecol 2004; 23(3): 267-271.

7. Wlaźlak E, Kluz T, Kociszewski J, Frachowicz K, Janowska M, Wlaźlak W et al.: The analysis of repeatability and reproducibility of bladder neck mobility measurements obtained during pelvic floor sonography performed introitally with 2D transvaginal probe. Ginekol Pol 2017; 88(7): 360-365.

8. Dresler MM, Kociszewski J, Wlaźlak E, Pędraszewski P, Trzeciak A, Surkont G: Repeatability and reproducibility of measurements of the suburethral tape location obtained in pelvic floor ultrasound performed with a transvaginal probe. J Ultrason 2017;17(69): 101-105.

9. Harms L, Emons G, Bader W, Lange R, Hilgers R, Viereck V: Funneling before and after anti-incontinence surgery - a prognostic indicator?

\section{Conclusions}

1. TVT position in relation to the pubic symphysis important for the cure of stress urinary incontinence.

2. Reduced TVT-pubic symphysis distance translates into shorter urethral funneling, but has no effects on funneling width in cured patients with persistent postoperative funneling.

\section{Conflict of interest}

The authors do not report any financial or personal connections with other persons or organizations, which might negatively affect the contents of this publication and/or claim authorship rights to this publication.

Part 2: tension-free vaginal tape. Int Urogynecol J Pelvic Floor Dysfunct 2007; 18(3): 289-294.

10. Kociszewski J, Rautenberg O, Perucchini D, Eberhard J, Geissbühler V, Hilgers R et al.: Tape functionality: sonographic tape characteristics and outcome after TVT incontinence surgery. Neurourol Urodyn 2008; 27(6): 485-490.

11. Kociszewski J, Rautenberg O, Kolben S, Eberhard J, Hilgers R, Viereck V: Tape functionality: position, change in shape, and outcome after TVT procedure - mid-term results. Int Urogynecol J 2010; 21(7): 795-800.

12. Lemos N, Korte JE, Iskander M, Freeman R, Arunkalaivanan A, Rizk D et al.: Center-by-center results of a multicenter prospective trial to determine the inter-observer correlation of the simplified POP-Q in describing pelvic organ prolapse. Int Urogynecol J 2012; 23(5): 579-584.

13. Chantarasorn V, Shek KL, Dietz HP: Sonographic appearance of transobturator slings: implications for function and dysfunction. Int Urogynecol J 2011; 22(4): 493-498.

14. Duckett J, Basu M, Papanikolaou N: Transperineal ultrasound to assess the effect of tension-free vaginal tape position on flow rates. Ultrasound Obstet Gynecol 2010; 36(3): 379-383.

15. Yang JM, Yang SH, Huang WC: Dynamic interaction involved in the tension-free vaginal tape obturator procedure. J Urol 2008; 180(5): 2081-2087.

16. Dietz HP, Mouritsen L, Ellis G, Wilson PD: How important is TVT location? Acta Obstet Gynecol Scand 2004; 83(10): 904-908.

17. Falconer C, Ekman-Ordeberg G, Malmström A, Ulmsten U: Clinical outcome and changes in connective tissue metabolism after intravaginal slingplasty in stress incontinent women. Int Urogynecol J Pelvic Floor Dysfunct 1996; 7(3): 133-137. 